TRANSACTIONS OF THE

AMERICAN MATHEMATICAL SOCIETY

Volume 355, Number 6, Pages 2183-2203

S 0002-9947(03)03158-1

Article electronically published on January 31, 2003

\title{
CENTRAL KÄHLER METRICS WITH NON-CONSTANT CENTRAL CURVATURE
}

\author{
ANDREW D. HWANG AND GIDEON MASCHLER
}

\begin{abstract}
The central curvature of a Riemannian metric is the determinant of its Ricci endomorphism, while the scalar curvature is its trace. A Kähler metric is called central if the gradient of its central curvature is a holomorphic vector field. Such metrics may be viewed as analogs of the extremal Kähler metrics defined by Calabi. In this work, central metrics of non-constant central curvature are constructed on various ruled surfaces, most notably the first Hirzebruch surface. This is achieved via the momentum construction of Hwang and Singer, a variant of an ansatz employed by Calabi (1979) and by Koiso and Sakane (1986). Non-existence, real-analyticity and positivity properties of central metrics arising in this ansatz are also established.
\end{abstract}

\section{INTRODUCTION}

Central Kähler metrics were investigated in [Ms2], where an extensive comparison was carried out with the extremal Kähler metrics of Calabi [C1], C2]. The latter are defined in terms of the scalar curvature, the former via the central curvature. Both are elementary symmetric functions of the Ricci endomorphism: the scalar curvature is its trace, while the central curvature is its determinant. The corresponding metric notion relates each curvature potential with the complex-analytic structure of the underlying manifold.

The main achievement of this work is the construction of central Kähler metrics with non-constant central curvature on various ruled surfaces, using the momentum construction, following $[\mathrm{HSn}$. Under appropriate geometric hypotheses, this ansatz converts the construction of a metric into a boundary value problem in one variable. The ansatz is equivalent to the one introduced by Calabi [C0], [C1], but is expressed in coordinates more convenient for our purposes. It should be noted that the boundary value problem for central metrics is more complicated than that arising for extremal ones, where a completely explicit solution may be obtained.

The spaces on which the momentum construction is defined include manifolds of the form $N=\mathbb{P}(L \oplus \mathcal{O})$, where $p: L \rightarrow M$ is a holomorphic Hermitian line bundle over a compact Kähler base manifold $M$, as well as the spaces obtained from such a ruled manifold by blowing down one or both of its ends to a lowerdimensional submanifold. In other words, the ansatz is set up on $S^{1}$-equivariant compactifications of $\mathbb{C}^{*}$-bundles. This paper contains structure theorems for central

Received by the editors November 1, 1999.

2000 Mathematics Subject Classification. Primary 53C55, 53C25.

The first author was supported in part by an NSERC Canada individual research grant.

The second author was partially supported by the Edmund Landau Center for research in Mathematical Analysis and Related Areas, sponsored by the Minerva Foundation (Germany). 
metrics in this general setting, and existence-related theorems in the case where $M$ is a compact Riemann surface.

Describing the latter first, let $k$ be the Euler number of the Riemann surface, and $n>0$ the Chern number of $L$. Our results suggest that the ansatz yields (nonconstant) central metrics exactly when $n<|k|$, with nowhere-vanishing central curvature. This is proven for every $n$ when the Euler number $k$ vanishes, i.e., when the base is a one-dimensional torus. For other values of the Euler number, special cases are proven. The following theorem summarizes what is demonstrated, with regards to existence as well as non-existence:

Theorem 1.1. The momentum construction yields biholomorphic isometry classes of real-analytic central metrics with non-constant central curvature, in Kähler classes of the following spaces:

- The first Hirzebruch surface $\mathbb{P}(\mathcal{O}(1) \oplus \mathcal{O}) \rightarrow \mathbb{P}^{1}$, with the metrics having positive central curvature and a positive definite Ricci tensor.

- Any ruled surface $\mathbb{P}(L \oplus \mathcal{O}) \rightarrow \Sigma_{2}$, where $L$ has Chern number one, and $\Sigma_{2}$ is a compact Riemann surface of genus two, with the metrics having negative central curvature.

The momentum construction yields no central metrics on the second Hirzebruch surface, and none with non-constant central curvature on any ruled surface $\mathbb{P}(L \oplus \mathcal{O})$ over a compact Riemann surface of genus one.

Our methods also work for other special choices of Chern number and Kähler class, and it is hoped that they can be extended to higher-dimensional KählerEinstein base spaces as well.

Turning to the structure theorems that hold in the above set-up, we note that the (non-constant) central metrics of the momentum construction are never extremal, and also demonstrate that if the base manifold is Fano, the central curvature of $g$ is positive if and only if its Ricci tensor is positive definite. Furthermore, there is a canonical local representation of the metric along a fiber, with respect to which it has a base and a vertical component. We show the following:

Theorem 1.2. If the vertical component of a central metric is of class $C^{2}$, then it is real-analytic.

See Theorem 3.6 for the precise statement. It follows from severe restrictions implied by centrality on the functions used in the construction of the solution metric. These also imply further non-existence results for a variety of higher-dimensional base spaces.

Section 2 summarizes the pertinent features of the momentum construction; details can be found in [HSn. Earlier forms of it appear in [C0], [KS1, KS2]. Many other works make use of the ansatz to construct metrics with various curvature properties, including $[\mathrm{Ki}],[\mathrm{Sk}],[\mathrm{H}],[\mathrm{G}], \overline{\mathrm{Mb}}], \mathrm{Sm}], \overline{\mathrm{LSn}}], \overline{\mathrm{PP}},[\mathrm{Tf}]$. In Section 3 the central curvature is expressed in terms of the data used in the ansatz. Boundary and integrability conditions for the resulting centrality equation, as well as the highly restrictive properties of the latter, are then pursued. Theorem 1.2 positivity, and non-extremality then follow from these considerations.

Section 4 is devoted to the proof of Theorem 1.1. After reviewing the ansatz for the case of a one-dimensional base, branching possibilities for real-analytic functions used in the construction of possible solution metrics are classified according to the 
topology. The final sections detail uniqueness and existence possibilities as deduced from the solvability of a pivotal nonlinear integrability condition.

The definition of a central Kähler metric below concludes this introduction. Let $(N, g)$ be a compact Kähler manifold of complex dimension $\ell$. Denoting its Kähler form by $\omega$ and its Ricci form by $\rho$, the central curvature function $C$ is defined by the equation

$$
\rho^{\wedge \ell}=C \omega^{\wedge \ell} .
$$

In other words, if one defines the Ricci endomorphism $\varrho$ via the metric (by "raising the second index" of $\rho$ with respect to $\omega$ ), then the central curvature is simply the complex determinant det $\varrho$. A central Kähler metric (of type $I$, in the terminology of [Ms2] $)$ is a metric for which the $(1,0)$-gradient vector field of $C$ is holomorphic.

\section{Salient Features of the Ansatz}

First, to describe the manifolds $N$ considered in this work we give the following brief review of the essential ingredients of the momentum construction. Its basic data are a holomorphic, Hermitian line bundle $p:(L, h) \rightarrow\left(M, \omega_{M}\right)$ over a compact connected Kähler manifold of dimension $l-1$, and an interval of real numbers that is here assumed to be of the form $[-T, T]$ for some $T>0$ (sufficiently small). The curvature form of $(L, h)$ is denoted $\gamma$, and the related $\omega_{M}$-associated endomorphism of $T^{1,0} M$ is denoted $B$. The manifold $N$ associated to these data is now taken to be either $\widehat{L}:=\mathbb{P}(L \oplus \mathbf{1}) \stackrel{p}{\rightarrow} M$ - the completion of the total space of $L$ - or else the space obtained from $\widehat{L}$ (if possible) by blowing down the zero section, the infinity section, or both, to submanifolds of higher codimension. Describing a space $N$ of the latter type in some more detail, there are natural identifications of $M$ with the zero and infinity sections of $\widehat{L}$, and if $D_{0}$ and $D_{\infty}$ denote the corresponding centers of blowing up in $N$, of respective complex codimensions $d_{0}$ and $d_{\infty}$, then there are projections from $M$ to $D_{0}$ and $D_{\infty}$. These may be constructed from a pair of holomorphic vector bundles $E_{0} \rightarrow D_{0}$ and $E_{\infty} \rightarrow D_{\infty}$ for which $\mathbb{P}\left(E_{0}\right) \simeq \mathbb{P}\left(E_{\infty}\right)$, together with respective dual tautological bundles. Non-trivial examples are given in [KS2], [FMS].

On $N$, a Kähler class $\Omega$ is constructed out of the momentum data by requiring that its restriction to the zero section of $N$ is $\left[\omega_{M}-T \gamma\right]$ and that the $\mathbb{P}^{1}$ fibres of $N$ (or their images under the blow-down map) have area $4 \pi T$. Consequently, the restriction of $\Omega$ to the infinity section of $N$ is $\left[\omega_{M}+T \gamma\right]$.

Remark 2.1. When $N=\widehat{L}$, it is assumed that the closed $(1,1)$-forms $\omega_{M} \pm T \gamma$ are positive. Otherwise they are required to be "suitably" nonnegative on $M$, in the sense that $\omega_{M}+T \gamma$ is the pullback of a Kähler form on $D_{0}$ and $\omega_{M}-T \gamma$ is the pullback of a Kähler form on $D_{\infty}$. In terms of the curvature endomorphism $B$, suitable nonnegativity means that the endomorphism $I-\tau B$ has nullity $d_{0}-1$ when $\tau=-T$, and nullity $d_{\infty}-1$ when $\tau=T$.

Given the above data, the momentum construction associates a smooth Kähler metric $g_{\varphi}$ on $N$ to each smooth ("profile") function $\varphi:[-T, T] \rightarrow \mathbb{R}$ that is positive on $(-T, T)$ and satisfies the boundary conditions

$$
\varphi( \pm T)=0, \quad \varphi^{\prime}( \pm T)=\mp 2 .
$$


The associated Kähler form, denoted $\omega_{\varphi}=\omega$, represents $\Omega$. The isometry class of $g_{\varphi}$ is determined by its restrictions to the zero and infinity sections, and by the profile $\varphi$. Distinct profiles do in fact yield distinct metrics.

Before describing the metric $g_{\varphi}$ and its geometry in more detail, we list further curvature specifications of the ansatz that reduce the curvature determination of the metric to an ordinary differential equation for $\varphi$. The base metric with Kähler form $\omega_{M}$ and the fibre metric $h$ are assumed to satisfy the following conditions:

(1) The eigenvalues of the curvature endomorphism $B$ are constant on $M$.

(2) The eigenvalues of the Ricci endomorphism $\varrho_{M}$ are constant on $M$.

(3) The endomorphisms $B$ and $\varrho_{M}$ are pointwise simultaneously diagonalizable.

The Metric and its Geometry. Since single-direction variations of the Kähler form $\omega_{M}$ will be considered in Section 4 it will be convenient to parameterize these by viewing $\omega_{M}$ as a multiple of some fixed Kähler form $\omega_{0}$. Thus we set $\omega_{M}=2 \pi a \omega_{0}$, for some positive real number $a$.

The following equation of top-dimensional forms in $M$ defines a function $Q$ on $[-T, T]$ via

$$
\left(\omega_{M}-x \gamma\right)^{\wedge(\ell-1)}=\frac{1}{a^{\ell-1}} Q(x) \omega_{M}^{\wedge(\ell-1)} .
$$

In other words, $Q(x)=a^{\ell-1} \operatorname{det}(I-x B)$. Under the curvature assumptions made above, $Q$ is a nonnegative polynomial function of degree at most $\ell-1$. It is nonvanishing in $(-T, T)$, and vanishes to order $d_{0}-1$ at $-T$ and to order $d_{\infty}-1$ at $T$. In particular, if $N=\widehat{L}$, then $Q$ is positive in $[-T, T]$. The Kähler forms $\omega_{M}-x \gamma$ all have the same Ricci form, denoted $\rho_{M}$. It is also useful to introduce the function

$$
\Psi:=\frac{1}{2 Q}(\varphi Q)^{\prime}=\frac{1}{2}\left(\varphi^{\prime}+\frac{\varphi}{Q} Q^{\prime}\right) .
$$

The latter is smooth since $\varphi$ and $Q$ are, and since its zeros cancel with its singularities at $x= \pm T$. By (2.1), it satisfies the boundary conditions

$$
\Psi(-T)=d_{0}, \quad \Psi(T)=-d_{\infty} .
$$

The open set $N^{\times}:=N \backslash\left(D_{0} \cup D_{\infty}\right)$ is the total space of a $\mathbb{C}^{*}$-bundle over $M$. At each point $z \in M$ there exist holomorphic coordinates $\left\{z^{\alpha}\right\}$ such that $z^{\alpha}(z)=0$ for all $\alpha$ and the components of $\omega$ and its Ricci form $\rho:=\rho_{\varphi}$ along the fibre over $z$ are given by the block matrices

$$
\left[g_{i \bar{\jmath}}\right]=\left[\begin{array}{cc}
\frac{1}{\varphi(\tau)} & 0 \\
0 & \left(\omega_{M}-\tau \gamma\right)_{\alpha \bar{\beta}}
\end{array}\right] ; \quad\left[\rho_{i \bar{\jmath}}\right]=\left[\begin{array}{cc}
-\frac{1}{\varphi(\tau)} \Psi^{\prime}(\tau) & 0 \\
0 & \left(\rho_{M}+\Psi(\tau) \gamma\right)_{\alpha \bar{\beta}}
\end{array}\right] .
$$

Here Greek indices run through $1, \ldots, \ell-1$, while the Roman ones run through $0, \ldots, \ell-1$. One can regard $\tau$ as the identity function on $(-T, T)$, with the above matrix-valued functions considered as living on $(-T, T) \times M$. A more direct description on $N$ is obtained from the following geometric presentation of the ansatz.

There is a holomorphic circle action on $N$, induced by scalar multiplication in the fibres of $L$. The moment map with respect to $\omega$ is a real-valued function $\tau$ whose image is $[-T, T]$, and the function $\varphi(\tau)$ is the length (squared) of the standard generator of the circle action. The gradient of $\tau$ with respect to $\omega$ is induced from

\footnotetext{
${ }^{\dagger}$ Actually $\varphi \circ \tau$, but the notation $\varphi(\tau)$ is compelling and useful.
} 
the Euler field on $\widehat{L}$. Note that holomorphy potentials that depend solely on $\tau$ are necessarily $\tau$-affine functions.

The symplectic reduction of $N$ at $\{\tau=x\}$ is $\left(M, \omega_{M}-x \gamma\right)$; it is often helpful to view $\omega_{M}-x \gamma$ as a (non-closed) form on $[-T, T] \times M$. This is in line with the positivity conditions assumed for $\omega_{M} \pm T \gamma$. Projecting $N$ to this space gives, over each point $(x, z) \in[-T, T] \times M$ with $-T<x<T$, a fiber which is a circle of length $2 \pi \sqrt{\varphi(x)}$. Together with the positivity of $\varphi$, this accounts for the first pair of boundary conditions in (2.1). The second pair ensures the smooth extendibility of the metric to the zero and infinity sections. Finally, up to a constant multiple, $Q(\tau) d \tau$ is the induced Duistermaat-Heckmann measure on $[-T, T]$, and the function $\varphi Q(\tau)$ is the volume density of $g_{\varphi}$ on $N$.

Remark 2.2. Note that the block-diagonality of the relations (2.3) holds only along a single fibre, with the off-diagonal terms eliminated by a particular choice of gauge; see [KS1 or $\mathrm{HSn}$ for the complete expressions. However, due to the algebraic nature of the central curvature, these relations are sufficient for its calculation.

Example 2.3. In the prototypical example, $M$ is a compact Kähler-Einstein manifold of dimension $\ell-1$ (or even a product of such spaces of varying dimensions), with $\omega_{0}$ now an indivisible and integral Kähler form which is a multiple of the first Chern class of $M$. Let $a$ and $T$ be positive real numbers, and $n$ a positive integer such that $a-n T>0$. The holomorphic Hermitian line bundle $(L, h)$ is chosen to have curvature form $\gamma=2 \pi n \omega_{0}$. Such a bundle always exists, and is unique if $M$ is simply-connected. The base metric is still $\omega_{M}=2 \pi a \omega_{0}$, and its Ricci form is $\rho_{M}=2 \pi k \omega_{0}$ for some integer $k$. The curvature and Ricci endomorphisms are multiples of the identity, with eigenvalues $n / a$ and $k / a$, respectively. The metric $\omega$ lives on the completion of $L$, and a quadratic profile satisfying (2.1) provides a simple example for which the $\mathbb{P}^{1}$ fibres of the induced metric are round spheres.

\section{Central Metrics in the Momentum Construction}

Fix a holomorphic Hermitian line bundle $p:(L, h) \rightarrow\left(M, \omega_{M}\right)$ and a momentum interval $[-T, T]$ as in Section 2. Let $\eta_{i}$ and $\kappa_{i}(1 \leq i \leq \ell-1)$ be the constant, not necessarily distinct eigenvalues of the curvature endomorphism $B$ and of the Ricci endomorphism $\varrho_{M}$, respectively, indexed with respect to a simultaneously diagonalizing eigenframe $\left\{e_{i}\right\}$.

3.1. The Central Curvature. The polynomial $Q$ is given by

$$
Q(x)=a^{\ell-1} \prod_{i=1}^{\ell-1}\left(1-\eta_{i} x\right) ;
$$

all its roots are real, exactly $d_{0}-1$ linear factors vanish at $-T$, and $d_{\infty}-1$ vanish at $T$. It will be of great use to introduce another polynomial function on $[-T, T]$ :

$$
R^{\prime}(x):=a^{\ell-1} \prod_{i=1}^{\ell-1}\left(\kappa_{i}+\eta_{i} x\right) .
$$

For each $x$, this polynomial is the determinant of the form $\rho_{M}+x \gamma$, regarded as an endomorphism via the Kähler form $\omega_{M}$. Its antiderivative will be of equal interest to us, as indicated by the prime notation. The polynomials $Q$ and $R^{\prime}$ obviously have the same degree, equal to the rank of the curvature endomorphism $B$. 
The central curvature $C=C(\tau)$ may be specified in the momentum construction. It is immediately calculable along a fibre of $N^{\times}$from relations (2.3):

$$
C(\tau)=\varphi\left(-\frac{1}{\varphi} \Psi^{\prime}(\tau)\right) \prod_{i=1}^{l-1}\left(\frac{1}{1-\tau \eta_{i}}\left(\kappa_{i}+\Psi(\tau) \eta_{i}\right)\right) .
$$

In terms of the functions just defined, it is given by

$$
C(\tau)=-\frac{1}{Q(\tau)} R^{\prime}(\Psi(\tau)) \Psi^{\prime}(\tau)=-\frac{(R \circ \Psi)^{\prime}}{Q}(\tau) .
$$

Because the central curvature is a scalar, and thus independent of the choice of the coordinate system, this equation holds globally on $N^{\times}$, hence on $N$ by continuity. The central curvature is "a function of $\tau$ alone". So, as noted previously, it is a holomorphy potential if and only if it is affine in $\tau$.

A priori, the right-hand side of (3.3) has poles where $Q$ vanishes. However, Lemma 3.1 below asserts that endpoint roots of $Q$ correspond to roots of $R^{\prime} \circ \Psi$. This is as it should be, since an arbitrary Kähler metric on $N$ has a smooth central curvature function.

Lemma 3.1. With notation as above, if $1+\eta_{i} T=0$, then $\kappa_{i}+\eta_{i} d_{0}=0$. Similarly, if $1-\eta_{i} T=0$, then $\kappa_{i}-\eta_{i} d_{\infty}=0$. More succinctly, if a linear factor of $Q$ vanishes at an endpoint $\pm T$, then the corresponding linear factor of $R^{\prime} \circ \Psi$ also vanishes at that endpoint.

Proof. In $T^{1,0} M$, choose the local frame $\left\{e_{i}\right\}_{i=1}^{\ell-1}$ so that the first $d_{0}-1$ vectors are annihilated by the projection $M \rightarrow D_{0}$. The vertical tangent bundle of this projection is exactly the null eigenbundle of $I+T B$. So if $1+\eta_{i} T=0$ for some $i$, then $D_{0}$ has codimension greater than 1 ; hence $M \rightarrow D_{0}$ is a non-trivial fibration. Its fibres are projective spaces of dimension $d_{0}-1$. The line bundle $L \rightarrow M$ is the tautological bundle over the projectivization of the normal bundle of $D_{0}$ in $N$. So its restriction to a fibre $F$ of $M \rightarrow D_{0}$ is $\mathcal{O}_{F}(-1)$. Therefore, if the restriction $\left.\omega_{M}\right|_{F}$ is $\hat{a}$ times the unit Fubini-Study form on $F$, then

$$
\eta_{i}=-\frac{1}{\hat{a}} \quad \text { for } 1 \leq i \leq d_{0}-1 .
$$

Since a fibre $F$ has anticanonical line bundle $\mathcal{O}\left(d_{0}\right)$,

$$
\kappa_{i}=\frac{d_{0}}{\hat{a}} \quad \text { for } 1 \leq i \leq d_{0}-1 .
$$

The lemma now follows by immediate substitution, and a similar argument for $D_{\infty}$

For brevity, the polynomial $R^{\prime}$ is called essentially non-vanishing if its only roots in the closed interval $\left[-d_{\infty}, d_{0}\right]$ are those accounted for by Lemma 3.1 If the image of $\Psi$ is equal to $\left[-d_{\infty}, d_{0}\right]$, then $R^{\prime}$ is essentially non-vanishing if and only if $\left(R^{\prime} \circ \Psi\right) / Q$ is non-vanishing on the closed interval $[-T, T]$.

3.2. Integrability Conditions. Given a smooth profile function with the correct nonnegativity behavior, construction of a central metric in this ansatz amounts to solving a second-order differential equation in $\varphi$, obtained by setting the central curvature to be affine,

$$
C(\tau)=b_{0}+b_{1} \tau
$$


and subject to the four boundary conditions (2.1). Using $\Psi$ and equation (3.3), this can be expressed as a system of two first-order equations in $\varphi$ and $\Psi$ :

$$
b_{0}+b_{1} \tau=-\frac{1}{Q(\tau)}(R \circ \Psi)^{\prime}(\tau), \quad \Psi=\frac{1}{2 Q}(\varphi Q)^{\prime},
$$

subject to the two boundary conditions (2.2), and to two further integrability conditions that will presently be described. Whenever the equation can be solved, these conditions are expected to determine the coefficients $b_{0}$ and $b_{1}$ uniquely.

Multiplying (3.3) by $-Q$ and integrating from $-T$ to $\tau$ gives

$$
P(\tau):=-\int_{-T}^{\tau} C(x) Q(x) d x=R \circ \Psi(\tau)-R\left(d_{0}\right),
$$

for an arbitrary smooth function $C$. The function $P$ will play an important role in what follows. It is a polynomial whenever $C$ is. In particular, if $g$ is a central metric with non-constant central curvature, $P$ has degree equal to $2+\operatorname{deg} Q$.

Setting $\tau=T$ gives the first integrability condition

$$
R\left(-d_{\infty}\right)-R\left(d_{0}\right)=\int_{d_{0}}^{-d_{\infty}} R^{\prime}=-\int_{-T}^{T} C Q .
$$

This gives a boundary condition on an arbitrary profile in terms of an integral involving the central curvature. For a central metric, equation (3.6) gives a linear condition on the constants $b_{0}$ and $b_{1}$, and will thus be referred to as the linear integrability condition. Since $P(-T)=0$, choosing $R\left(d_{0}\right)=0$ simplifies condition (3.6) to

$$
R\left(-d_{\infty}\right)=P(T) .
$$

For a central metric the right-hand side integral is indeed linear in $b_{0}$ and $b_{1}$, which leads to the possibility of eliminating one of these coefficients.

To get a second integrability condition, assume first that $R^{\prime}$ is non-vanishing on the image of $\Psi$ (except possibly at the endpoints). It follows that there is a single branch of $R^{-1}$ on the image of $\Psi$. Hence, still using the choice $R\left(d_{0}\right)=0$, one has $\Psi=R^{-1} \circ P$ on $[-T, T]$. Now, by the definition of $\Psi$ and the boundary conditions (2.1) on $\varphi$,

$$
2 \int_{-T}^{T} Q \Psi=(\varphi Q)(T)-(\varphi Q)(-T)=0 .
$$

Substituting for $\Psi$ gives the second integrability condition:

$$
\int_{-T}^{T} Q \cdot\left(R^{-1} \circ P\right)=0 .
$$

This condition again holds for arbitrary profile functions. When the metric $g$ is central, it will later be shown that it is not only smooth but in fact (vertically) real-analytic, and that the domain of $R$ can be split into at most two intervals on which $R$ is monotone. For the case of two intervals, $R^{-1}$ is composed of two branches $R_{I}^{-1}$ and $R_{I I}^{-1}$ that patch smoothly at a point $\tau_{0} \in(-1,1)$, and then (3.8) reads

$$
\int_{-T}^{\tau_{0}} Q \cdot\left(R_{I}^{-1} \circ P\right)+\int_{\tau_{0}}^{T} Q \cdot\left(R_{I I}^{-1} \circ P\right)=0 .
$$


The point $\tau_{0}$ will be characterized in the next two subsections as the unique one for which $\left(R^{\prime} \circ \Psi\right)\left(\tau_{0}\right)=0$.

Since equation (3.8) involves the inversion of $R$, it is relatively difficult to analyze. The appearance of $R^{-1}$ prevents it, in general, from being linear in $b_{0}$ and $b_{1}$. Henceforth it will be referred to as the nonlinear integrability condition.

3.3. Implications of the Linearity of the Central Curvature. So far, the metrics arising from the momentum construction have been assumed to be smooth. Unless the profile function is given explicitly, this may be difficult to verify in general. It will thus be useful to broaden the scope of allowed profiles. Assume $N$ admits a not necessarily smooth central Kähler metric $g_{\varphi}$ for which $\varphi$ is of class $C^{2}$ with $g_{M}$ smooth. With such assumptions, equation (3.4) is still well-defined, and since $C(\tau)$ is affine, implies the following:

Proposition 3.2. For any central metric arising from the momentum construction with a profile of class $C^{2}$, and with central curvature not identically zero and with $g_{M}$ smooth, exactly one of the following possibilities holds.

- The central curvature and $\Psi^{\prime}$ do not vanish anywhere in $[-T, T]$, and $R^{\prime}$ is essentially non-vanishing in $\left[-d_{\infty}, d_{0}\right]$.

- The central curvature and $\Psi^{\prime}$ both vanish with multiplicity one on the same unique value of $\tau$ in $[-T, T]$, and $R^{\prime}$ is essentially non-vanishing on $\left[-d_{\infty}\right.$, $d_{0}$ ] (or even on the image of $\Psi$ ).

- The central curvature vanishes for a unique value of $\tau$ in $[-T, T]$, while $R^{\prime}$ has a unique linear factor with a zero in $\left[d_{\infty}, d_{0}\right]$ besides possibly those vanishing at the endpoints by Lemma [3.1, and $\Psi^{\prime}$ is nowhere vanishing on $[-T, T]$.

It is implicitly understood above that for non-smooth functions, the multiplicity of vanishing is defined ad hoc to be one more than the number of well-defined vanishing derivatives at the point.

Remark 3.3. Note that since in any case $R^{\prime} \circ \Psi$ vanishes in at most one point of $(-T, T)$, this immediately rules out existence of central metrics on many spaces. In other words, by the third possibility above, if $R^{\prime}$ has more than one root in $\left(-d_{\infty}, d_{0}\right)$, then $N$ does not admit a central metric arising from the momentum construction. This happens, for example, if $M$ is a Ricci-flat manifold of dimension at least two and $L$ is a line bundle with non-degenerate curvature.

Remark 3.4. Since our interest lies in non-constant central curvature, it was assumed above that it does not vanish identically by excluding $R^{\prime} \equiv 0$. The latter leads to $L$ with degenerate curvature and $M$ a base also of vanishing central curvature. The other possibility for $C=0$, namely $\Psi \equiv c$ on $[-T, T]$, cannot occur. For otherwise, by definition of $\Psi$ and the boundary conditions (2.1), $0=\left.(\varphi Q)\right|_{-T} ^{T}=2 c \int_{-T}^{T} Q ;$ so $c=0$. Thus $\varphi Q$ is constant, hence also identically zero.

Remark 3.5. That $\Psi^{\prime}<0$ in $(-T, T)$ follows from assuming that $\Psi$ has image equal to $\left[-d_{\infty}, d_{0}\right]$ will become apparent once we prove in the next subsection that centrality of the metric implies that it is (vertically) real-analytic. This indeed follows, since $\Psi$ would then be real-analytic with absolute extrema at the endpoints and with at most one critical point (counting multiplicity), which is not an endpoint. 
Consequently $\Psi^{\prime}$ has no such zeros. Hence, if $\Psi^{\prime}$ is nowhere vanishing in $(-T, T)$, then $\Psi$ decreases from $d_{0}$ to $-d_{\infty}$.

Again using real-analyticity, the second possibility of the proposition cannot occur at an interior point if neither end of $N$ is a divisor: if $C(\tau)$ and $\Psi^{\prime}$ change sign exactly once at an interior point, this implies that $\Psi$ increases near one of the endpoints and consequently has image larger than $\left[-d_{\infty}, d_{0}\right]$. By the intermediate value theorem, its value at that endpoint is attained in the open interval $(-T, T)$, and if $R^{\prime}$ vanished on it, as must happen with lower-dimensional ends, the allowed number of zeros of $C$ would be exceeded.

3.4. Smoothness Considerations for Central Metrics. This subsection is devoted to showing that centrality implies real-analyticity, at least for the vertical component of a metric arising from the momentum construction, even in a situation where the corresponding profile is not known to be smooth or nonnegative.

In fact, as noted previously, as long as $R$ is invertible, $\Psi=R^{-1} \circ P$ defines a realanalytic function on $[-T, T]$, and therefore, if $\Psi$ solves the centrality equations (3.4), $\varphi$ and consequently the vertical part of $g$ are also real-analytic. The following renders Theorem 1.2 .

Theorem 3.6. Let $\varphi$ be a function of class $C^{2}$ satisfying the boundary conditions (2.1), and which (together with a related function $\Psi$ ) gives a solution to equations (3.4). If $R^{\prime}$ is not identically zero, then $\varphi$ is real-analytic. In addition, $\varphi$ is positive on $(-T, T)$.

Proof. As mentioned above, to prove $\varphi$ is real-analytic, it is enough to show $\Psi$ is. If $R^{\prime}$ is not identically zero, then its roots are discrete, and Proposition 3.2 implies that the image of $\Psi$ can be partitioned into at most two intervals on which $R^{\prime}$ does not vanish, except perhaps at their endpoints. On each such interval, there is a continuous branch of $R^{-1}$ (which is real-analytic except perhaps at the endpoints). So, as before, $\Psi=R^{-1} \circ P$ is real-analytic except possibly at a point $\tau_{0}$ with $\left[\left(R^{\prime} \circ \Psi\right) / Q\right]\left(\tau_{0}\right)=0$.

Since $\Psi$ is of type $C^{1}$ by assumption, $\Psi^{\prime}$ is well-defined. If there is such a $\tau_{0} \in(-1,1)$, then the third possibility in Proposition 3.2 is satisfied, so that $\Psi^{\prime}$ is non-vanishing on $[-T, T]$. Write equation (3.3) using the inverse function theorem as

$$
\Psi^{\prime}(\tau)=-\frac{C(\tau) Q(\tau)}{\left(R^{\prime} \circ \Psi\right)(\tau)}=\left(\left(R^{-1}\right)^{\prime} \circ P\right)(\tau) \cdot P^{\prime}(\tau) .
$$

The order of vanishing of $C Q$ at $\tau_{0}$ must then necessarily equal the order of vanishing of $R^{\prime}$ at $\Psi\left(\tau_{0}\right)$; call this number $\delta$. Of course, $\delta=1$. By (3.5), there exist polynomials $P_{0}$ and $R_{0}$, not vanishing at $\tau_{0}$ and $\Psi\left(\tau_{0}\right)$, respectively, such that

$$
P(x)=P\left(\tau_{0}\right)+\left(x-\tau_{0}\right)^{\delta+1} P_{0}(x), \quad R(y)=R\left(\Psi\left(\tau_{0}\right)\right)+\left(y-\Psi\left(\tau_{0}\right)\right)^{\delta+1} R_{0}(y) .
$$

Since the powers in these expressions are equal, there exist branches of $R^{-1}$ that can be patched to make $R^{-1} \circ P$ real-analytic at $\tau_{0}$, and those can thus be used to define $\Psi$ on $[-T, T]$.

Regarding positivity, by definition $(\varphi Q)^{\prime}=2 Q \Psi$, while $\Psi(-T)=d_{0}>0>$ $-d_{\infty}=\Psi(T)$ by the boundary conditions on $\varphi$. Using the real-analyticity, it follows that $\Psi$ vanishes an odd number of times in $(-T, T)$, counting multiplicity. If $\Psi$ had three or more zeros, then it would have at least two critical points, contradicting Proposition 3.2. Thus $\Psi$ vanishes exactly once, and so $\varphi Q$ has exactly one critical 
point. Since $\varphi$ is positive near $\pm T$ and $Q$ is positive on $(-T, T)$, the product is positive on $(-T, T)$, as desired.

3.5. Positivity Conditions. In this subsection the positivity of the central curvature is related with the positivity of $c_{1}(N)$. This situation is an example of the first possibility listed in Proposition 3.2 Note the following related though simpler conclusion: since 0 is a value of $\Psi$, if the sign of the central curvature of $g_{\varphi}$ is fixed, it is the same as the sign of $\prod_{i} \kappa_{i}$, the central curvature of $g_{M}$.

The following is the positivity result alluded to in the introduction, and should be considered a consequence of Proposition 3.2. It is conceivable that it may be proved without alluding to the particularities of the momentum construction.

Theorem 3.7. Let $N$ be a (possibly blown-down) ruled manifold as above, and assume $N$ admits a central Kähler metric $g$ arising from the momentum construction. The following are then equivalent:

- the Ricci curvature of $g$ is positive;

- the base space $M$ is Fano and the central curvature of $g$ is positive.

In particular, the latter conditions imply $N$ is Fano.

Proof. Though positivity of the Ricci tensor obviously implies positivity of the central curvature, it is instructive to prove this in the framework of the ansatz.

Assume the Ricci curvature of $g$ is positive. Then, by the Kähler and Ricci form expressions (2.3),$\Psi^{\prime}<0$ on $(-T, T)$, since $\varphi$ is positive there. Turning to the endpoints, the ratio of the vertical components of the Ricci and Kähler forms is $-\Psi^{\prime}( \pm T)$. Since the ansatz guarantees that the Kähler form extends positively $[\mathrm{H}]$, [HSn, KS1], and so does the Ricci form by assumption, $\Psi^{\prime}$ is negative at the endpoints.

Taking $\left\{e_{i}\right\}$ to be a simultaneously diagonalizing eigenframe as in the beginning of the section, expressions (2.3) also show that $\kappa_{i}+\Psi \eta_{i} \geq 0$ on $[-T, T]$, with equality at most at $\pm T$ exactly when the corresponding linear factor of $Q$ vanishes at $\pm T$. This follows by Lemma 3.1, and by the positivity of the limiting ratio of the components of the Kähler and Ricci forms in the non-blown-down directions $e_{i}$ at $\pm T$.

Thus expression (3.3) for the central curvature is positive, and by l'Hôpital's rule,

$$
\lim _{\tau \rightarrow \pm T} \frac{R^{\prime} \circ \Psi(\tau)}{Q(\tau)}>0
$$

Hence the central curvature is positive even on the ends of $N$.

Since $\varrho_{M}$ has constant eigenvalues, $M$ is Fano if and only if $\kappa_{i}>0$ for all $i$. Since 0 is a value of $\Psi$, we have $0<k_{i}=k_{i}+\Psi(\hat{\tau}) n_{i}$ at some point $\hat{\tau} \in(-T, T)$; so $M$ is indeed Fano.

Conversely, assume $\kappa_{i}>0$ for all $i$, and that

$$
C(\tau)=-\Psi^{\prime}(\tau) \prod_{i} \frac{\kappa_{i}+\Psi(\tau) \eta_{i}}{1-\tau \eta_{i}}>0 \quad \text { on }[-T, T],
$$

with the expression understood at the endpoints in a limiting sense. Since $Q$ is positive in $(-T, T)$, the product of the linear factors in the denominator is positive there. By Proposition 3.2 and Remark 3.5, we see that $\Psi^{\prime}<0$ as well, even on $[-T, T]$. Finally, each linear factor in the numerator is nowhere vanishing and 
positive where $\Psi$ vanishes, hence necessarily positive on $(-T, T)$. Consequently, by expressions (2.3) the Ricci form is positive except perhaps at the ends. But if $\kappa_{i}+\Psi( \pm T) \eta_{i}=0$ without $1 \mp T \eta_{i}=0$, the limiting value (3.10) of $C$ will be zero and not positive. Thus for each direction $e_{i}$ that is not blown-down we have $\kappa_{i}+\Psi( \pm T) \eta_{i}>0$. Thus the Ricci curvature of $g$ is positive.

3.6. Central versus Extremal Metrics. Before demonstrating the existence of central metrics in the examples of the next section, it is shown below that such metrics are not, in any case, extremal.

Proposition 3.8. Let $g$ be a central Kähler metric arising from the momentum construction. If its central curvature is non-constant, then $g$ is not extremal in the sense of Calabi.

Proof. In this ansatz, the scalar curvature of an arbitrary metric depends only on $\tau$, and it was previously noted that the only purely $\tau$-dependent holomorphy potentials are affine. However, if the scalar curvature of a metric is polynomial in $\tau$, then its profile $\varphi$ is rational in $\tau$; this can be verified from (2.3) by computing the trace of the Ricci endomorphism, see [HSn].

It therefore suffices to show that a central metric of non-constant central curvature is associated to a non-rational profile, and this is clear from the following observations. The polynomials $Q$ and $R^{\prime}$ have the same degree. So if $C$ is a nonconstant affine function, then the polynomials $P$ and $R$ do not have the same degree. Consequently, $\Psi=R^{-1} \circ P$ is not a rational function, and therefore the profile $\varphi$ is not rational either.

\section{The Case of a One-Dimensional Base}

This section is devoted to a concrete and detailed investigation of equations (3.4) and their integrability conditions, in the case where $M$ is a compact Riemann surface of genus $p$, and $N=\widehat{L}=\mathbb{P}(L \oplus \mathcal{O})$ is a ruled surface.

4.1. The Central Equation and the Integrability Conditions. The ansatz is reviewed below in the following special setting. It is enough to consider metrics up to homothety, hence set $T=1$. The formulas will be written in terms of the Euler number $k$ of $M$ and the Chern number $n$ of $L$, as in Example 2.3] rather than by means of $\kappa=k / a$ and $\eta=n / a$. Here $a$ parameterizes the base Kähler form via $\omega_{M}=2 \pi a \omega_{0}$, where $\omega_{0}$ is indivisible and integral. Since $T$ is fixed, $a$ also parameterizes the Kähler classes on $N$, under the restriction $a>|n|$. The freedom in the choice of sign for $n$ is eliminated by declaring it nonnegative. It is in fact positive, since our interest lies in spaces which may admit metrics of non-constant central curvature, and this cannot happen for $n=0$ (see [Ms2]). The base Ricci form is

$$
\rho_{M}=2 \pi k \omega_{0}=\frac{k}{a} \omega_{M}
$$

with $k=2-2 p$ the Euler number of the surface. The curvature form of $L$ is

$$
\gamma=2 \pi n \omega_{0}=\frac{n}{a} \omega_{M},
$$

and the Duistermaat-Heckman measure computes, from the ratio of areas of sections with respect to $\omega_{M}-\tau \gamma$ and $\omega_{M}$, to be

$$
\left(1-\tau \frac{n}{a}\right) d \tau=\frac{Q}{a} d \tau
$$


The centrality equation in (3.4) then reads

$$
C(\tau)=-\frac{1}{a-n \tau}\left(k \Psi+\frac{n}{2} \Psi^{2}\right)^{\prime}=b_{0}+b_{1} \tau .
$$

The function $R^{\prime}$ of the previous section is $R^{\prime}(x)=k+n x$. So taking $R(x)=k x+$ $\left(n x^{2}\right) / 2-(k+n / 2)$, the convenient antiderivative boundary value $R\left(d_{0}\right)=R(1)=0$ holds. Thus equation (3.5) reads

$$
P(\tau)=-\int_{-1}^{\tau}\left(b_{0}+b_{1} x\right)(a-n x) d x=k \Psi(\tau)+\frac{n}{2} \Psi^{2}(\tau)-\left(k+\frac{n}{2}\right)=(R \circ \Psi)(\tau) .
$$

The boundary conditions (2.2) on $\Psi$ are

$$
\Psi( \pm 1)=\mp 1 .
$$

For the linear integrability condition (3.7), one has

$$
P(1)=-\left(2 b_{0} a-2 \frac{b_{1} n}{3}\right)=-2 k=R(-1),
$$

which in turn is used to eliminate $b_{0}$ :

$$
b_{0}=\frac{b_{1} n}{3 a}+\frac{k}{a} .
$$

Substituting this relation gives a useful form for $P(\tau)$. A short computation gives

$$
P(\tau)=-(\tau+1)\left(k-\frac{k n}{2 a}(\tau-1)+b_{1}(\tau-1)\left(-\frac{n}{3} \tau-\left(\frac{n^{2}}{6 a}-\frac{a}{2}\right)\right)\right) .
$$

Turning to the nonlinear integrability condition, it is necessary to understand whether $R$ is invertible. In the concrete setting at hand one can compute $\Psi$ directly by completing the square in the right-hand side of (4.2):

$$
P(\tau)=\frac{n}{2}\left(\left(\Psi+\frac{k}{n}\right)^{2}(\tau)-\left(\frac{k}{n}+1\right)^{2}\right) .
$$

It follows that the branches of $\Psi$ are of the form

$$
\Psi(\tau)=-\frac{k}{n} \pm \sqrt{\frac{2 P(\tau)}{n}+\left(\frac{k}{n}+1\right)^{2}} .
$$

In other words, the branches of $R^{-1}$ are $R^{-1}(x)=-k / n \pm \sqrt{(2 / n) x+(k / n+1)^{2}}$. Recall that $R$ is invertible in $[-1,1]$ if $R^{\prime}$ is nonzero in $(-1,1)$. But $R^{\prime}=0$ at $x=-k / n$; so if $|-k / n| \geq 1$, only one of the branches above is used. Otherwise it is necessary to patch both at this interior point. The various possibilities will be listed in detail in the next subsection. We conclude by stating the nonlinear integrability condition (3.8) (for a single branch):

$$
0=\int_{-1}^{1}(a-n \tau)\left(-\frac{k}{n} \pm \sqrt{\frac{2}{n} P(\tau)+\left(\frac{k}{n}+1\right)^{2}}\right) d \tau
$$


Possible Choices for $R^{-1}$. The choice of branch of the inverse of $R$ must correspond both to the choice of antiderivative and to the resulting linear integrability condition:

$$
\begin{aligned}
& \Psi(-T)=d_{0}=R^{-1} P(-T), \\
& \Psi(T)=-d_{\infty}=R^{-1} P(T) .
\end{aligned}
$$

Since the left-hand side is \pm 1 , and the right-hand side computes to $-k / n \pm|k / n \pm 1|$, it follows that the topology of $N$ dictates the allowed branches near each endpoint, according to Table 1:

TABLE 1 . Topology and branches of $R^{-1}$ at endpoints

\begin{tabular}{|r||c|c|}
\hline Branch : & $\tau=-1$ & $\tau=1$ \\
\hline \hline$k \leq-n$ & $-\sqrt{ }$ & $-\sqrt{ }$ \\
\hline$k<|n|$ & $+\sqrt{ }$ & $-\sqrt{ }$ \\
\hline$k \geq n$ & $+\sqrt{ }$ & $+\sqrt{ }$ \\
\hline
\end{tabular}

Note that the second possibility involving a patch of branches cannot occur when $|k|=n$, since $k$ cannot be equal to both $n$ and $-n$ for $n \neq 0$.

The Nonlinear Integrability Condition: Explicit Form, Uniqueness and Existence Considerations. Combining (4.4) with (4.6), the right-hand side of the nonlinear integrability condition (for one branch) may be written in the form

$$
I\left(b_{1}\right):=\int_{-1}^{1}(a-n \tau) \cdot\left(-\frac{k}{n} \pm \sqrt{f_{1}(\tau) b_{1}+f_{2}(\tau)}\right) d \tau,
$$

where

$$
\begin{aligned}
f_{1}(\tau) & :=\frac{2}{n}\left(\tau^{2}-1\right)\left(\frac{n}{3} \tau+\left(\frac{n^{2}}{6 a}-\frac{a}{2}\right)\right), \\
f_{2}(\tau) & :=\frac{k}{a} \tau^{2}-\frac{2 k}{n} \tau-\frac{k}{a}-\frac{2 k}{n}+\left(\frac{k}{n}+1\right)^{2} .
\end{aligned}
$$

For the case of two branches, the positive branch is to be integrated in $\left[-T, \tau_{0}\right]$, and the negative one in $\left[\tau_{0}, T\right]$, with $\tau_{0}$ as in the previous section.

The coefficient $f_{1}$ does not depend on $k$. Its quadratic factor is non-positive for $-1 \leq \tau \leq 1$, and one can check that for $a>n$ its linear factor is negative in the closed interval. Therefore $f_{1}$ is nonnegative for $-1 \leq \tau \leq 1$, and vanishes only at the end points.

The free term $f_{2}$ is, of course, quadratic, and one can easily check that it is nonnegative, decreasing for $k>0$, increasing for $k<0$ and equal to the constant 1 when $k=0$. When $|k / n|=1, f_{2}$ factors to:

$$
\begin{array}{ll}
\frac{k}{a}(\tau-1)(\tau+1-2 a / k) & k>0, \\
\frac{k}{a}(\tau+1)(\tau-1+2 a / k) & k<0 .
\end{array}
$$


The function $I\left(b_{1}\right)$ is real-analytic on its domain, which is a half-closed infinite interval. On its interior, its derivative equals (for positive square root)

$$
I^{\prime}\left(b_{1}\right)=\frac{1}{2} \int_{-1}^{1}(a-n \tau) \cdot\left(f_{1}(\tau) b_{1}+f_{2}(\tau)\right)^{-\frac{1}{2}} f_{1}(\tau) d \tau,
$$

which is positive. Hence

Proposition 4.1. There is at most one $b_{1}$ in the domain of $I$ for which $I\left(b_{1}\right)=0$.

This ensures uniqueness of the metrics. The first step in the existence proof is the evaluation of $I(0)$ in the single branch case:

$$
I(0)=\frac{a}{3 k n}\left(-6 k^{2} \pm \frac{1}{n}\left(|k+n|^{3}-|k-n|^{3}\right)\right)= \pm \frac{2 n a}{3 k} .
$$

The sign of this expression depends on $k$. To verify that the nonlinear integrability condition is satisfied, it is enough to find a value of $b_{1}$ for which the sign of $I\left(b_{1}\right)$ is the reverse of the sign of $I(0)$. Continuity of $I$ will then ensure that the condition is achieved for some other intermediate value of $b_{1}$.

The remaining existence considerations are now separated into the different cases, with the following common strategy described first for the one-branch case. The endpoint of the semi-infinite interval consisting of the domain of $I$ is a negative number $b$ on which (the monotone function) $I$ achieves its extremum. In all cases $b$, and then $I(b)$, are either evaluated or estimated. In some cases the latter is compared with $I(0)$. For the estimation of $b$ one uses various characterizations of it. It is the maximum of $-f_{2} / f_{1}$, as well as the unique value of $b_{1}$ on which the radicand has a double root at a point $\tau_{0} \in[-1,1]$. Relating $\tau_{0}$ with $b$ and understanding the former is what allows for the determination of the latter. Moreover, the fact that $\tau_{0}$ is a double root means that the radicand simplifies, and consequently that $I(b)$ can, in principle, be evaluated explicitly. However, determining $\tau_{0}$ amounts to solving an equation of degree three, which yields expressions that are difficult to handle in full generality. We therefore present special cases in which evaluating $\tau_{0}$ is easier, or else it may be estimated.

The root $\tau_{0}$ occurs at a point for which $\left(R^{\prime} \circ \Psi\right)\left(\tau_{0}\right)=0$. In this sense the point considered in the proof of Theorem 3.6 provides an example. Indeed, turning to the two-branch case, such $\tau_{0}$ appears explicitly as a boundary value of both integrals comprising condition (3.9). In a special case that will be considered later, $\tau_{0}$ will remain a critical point of the radicand for any value of $b_{1}$. In that case, condition (3.9) may be written as $I_{+}\left(b_{1}\right)+I_{-}\left(b_{1}\right)=0$, with $I_{+}$and $I_{-}$the monotone functions corresponding to the two branches. The value $b$ may be defined as the (unique) common extremum of both. It also satisfies the characterizations given above and can be related similarly to $\tau_{0}$. The main conceptual difference from the one-branch case is that if a central metric exists, then the coefficient $b_{1}$ of its central curvature must equal $b$. This follows from the uniqueness of $\tau_{0}$ (and of $b$ ) as guaranteed in Theorem [3.6. the opposite monotonicity of $I_{+}$and $I_{-}$, and the requirement that $\Psi$ remains smooth. Thus the interest lies not in the sign of $I_{+}(b)+I_{-}(b)$, but in whether or not it vanishes. If it is not, then existence is obstructed.

In conclusion, we rephrase the geometric significance of $b$ and $\tau_{0}$ as follows. There is an extremal solution candidate $\Psi=\Psi_{b}$, which solves the first of equations (3.4). Its associated central curvature vanishes at a point $\tau_{0}$ which is determined by the topology via $\Psi_{b}\left(\tau_{0}\right)=-k / n$. It is not a solution to the full system, since it turns 
out that the nonlinear integrability condition forbids the vanishing of the central curvature. However, the claim that there are solutions exactly when $n<|k|$ may be translated into the statement that whenever $\Psi_{b}\left(\tau_{0}\right)$ lies outside the range $\left[-d_{\infty}, d_{0}\right]$, $\Psi_{b}$ can be adjusted to yield a solution with nowhere-vanishing central curvature.

\subsection{The Second Hirzebruch Surface.}

A Lower Bound on $I\left(b_{1}\right)$, for $|k|=n$. This subsection treats the case $|k|=n$. Since the formula for $f_{2}$ changes with the sign of $k$, the computations will be carried out only for $k>0$, and then briefly indicated for negative $k$. Now $k>0$ means of course $k=2$, and therefore $n=2$. Thus the following result is proved for the second Hirzebruch surface:

Theorem 4.2. The momentum construction yields no central metrics in (any Kähler class of) the second Hirzebruch surface.

Although the ansatz is used explicitly in the proof, it may turn out to be extraneous in the statement of the result.

Proof. The domain of $I\left(b_{1}\right)$ is limited by the requirement that the radicand remain nonnegative. This amounts to requiring $b_{1} \geq-f_{2}(\tau) / f_{1}(\tau)$ in $(-1,1)$, since $f_{1}(\tau)$ is positive there. At the endpoints, where $f_{1}$ vanishes, $f_{2}(\tau)$ is nonnegative, and so the square root is well-defined there for every value of $b_{1}$. Hence, to find where $I$ achieves its minimum, it is enough to find the maximum value $b$ of $h(\tau):=f_{2}(\tau) /\left(-f_{1}(\tau)\right)$, which may occur as a limiting value at an endpoint of $[-1,1]$.

The function $h$ simplifies to

$$
h(\tau)=\frac{\frac{2}{a}(\tau-1)(\tau-(a-1))}{\left(1-\tau^{2}\right)\left(\frac{2}{3} \tau+\left(\frac{4}{6 a}-\frac{a}{2}\right)\right)}=-\frac{12(\tau-(a-1))}{(\tau+1)\left(4 a \tau+4-3 a^{2}\right)} .
$$

A calculation shows that the critical points of $h$ are $3 a / 2-2$ and $a / 2$, both larger than 1 since $a>2$. Hence the best upper bound for $h$ in $(-1,1)$ is its limiting value at one of the endpoints. One can check that the limit value at $\tau=-1$ is $-\infty$. The maximum $b$ therefore occurs at $\tau=\tau_{0}=1$, and its value is

$$
\lim _{\tau \rightarrow 1^{-}} h(\tau)=-\frac{12(2-a)}{2\left(4 a+4-3 a^{2}\right)}=-\frac{6}{3 a+2} .
$$

Hence $I\left(b_{1}\right)$ is well-defined, so long as $b_{1} \geq-6 /(3 a+2)$.

Next, since $f_{2}$ vanishes at $\tau=1$ for $k=n=2$, the radicand factors once there. Substituting $b=-6 /(3 a+2)$ for $b_{1}$ gives, by the preceding discussion, a radicand vanishing even to second order at $\tau=1$. A computation gives:

$$
f_{1}(\tau) b+f_{2}(\tau)=f_{1}(\tau) \frac{-6}{3 a+2}+f_{2}(\tau)=\frac{1}{3 a+2}(\tau-1)^{2}(-4 \tau+3 a-2) .
$$

The integral $I(b)$ is computable. The integrand has a summand containing the radical. This summand simplifies as $\tau-1$ factors out of the radicand:

$$
\begin{aligned}
& \int_{-1}^{1}(a-2 \tau) \sqrt{\frac{1}{3 a+2}(\tau-1)^{2}(-4 \tau+3 a-2)} d \tau \\
= & \frac{1}{\sqrt{3 a+2}} \int_{-1}^{1}\left(2 \tau^{2}-(a+2) \tau+a\right) \sqrt{-4 \tau+3 a-2} d \tau .
\end{aligned}
$$

Making the substitution

$$
u=-4 \tau+3 a-2
$$


gives an integral which computes to

$$
\begin{gathered}
\frac{1}{112}(3 a+2)^{3}\left(1-\left(\frac{3 a-6}{3 a+2}\right)^{\frac{7}{2}}\right)+\frac{1}{20}(2-a)(3 a+2)^{2}\left(1-\left(\frac{3 a-6}{3 a+2}\right)^{\frac{5}{2}}\right) \\
+\frac{1}{16}(a-2)^{2}(3 a+2)\left(1-\left(\frac{3 a-6}{3 a+2}\right)^{\frac{3}{2}}\right) .
\end{gathered}
$$

This is to be compared with the summand of the integrand in $I(b)$ which does not involve the radical, i.e., with $\int_{-1}^{1}(a-n \tau)(k / n) d \tau=2 a k / n=2 a$. For the borderline value $a=2$, one gets

$$
\frac{8^{3}}{112} \simeq 4.57>4=2 a
$$

One can similarly check explicitly that this holds also for other values $a>2$. To proceed in making an estimate valid for all Kähler classes, the term

$$
-\sqrt{(3 a-6) /(3 a+2)}=-\sqrt{1-(8 /(3 a+2))}
$$

is replaced everywhere by the smaller quantity

$$
-(1-(4 /(3 a+2)))=-(3 a-2) /(3 a+2)
$$

(using $-\sqrt{1-x}>-(1-x / 2)$ for $x>0$ ). Doing this and subtracting $2 a$, one sees that $I\left(b_{1}\right)$ is larger than a quantity which computes to

$$
\frac{4}{35} \frac{315 a^{4}+2576 a^{2}+272+420 a^{3}+112 a}{(3 a+2)^{4}} .
$$

This expression is certainly positive for any $a>2$. Since $I\left(b_{1}\right)$ is increasing, it is therefore everywhere positive, and the nonlinear integrability condition is never satisfied. This completes the proof.

The Case $-k=n$. Similarly, when $k<0$, and $-k=n, h$ has roots $a / n$ and $2+3 a / n$, both larger than 1 , and its maximum in this case will be the limiting value when $\tau=-1$, and equals $-3 n /(3 a-n)$. With this value the radicand is

$$
f_{1}(\tau) \frac{-3 n}{(3 a-n)}+f_{2}(\tau)=-\frac{1}{3 a-n}(\tau+1)^{2}(2 \tau n-3 a-n) .
$$

The radical-summand of $I\left(b_{1}\right)$ is

$$
\begin{aligned}
&-1 / 35 \frac{3 a^{3}+11 a^{2} n-55 a n^{2}+17 n^{3}}{n^{2}} \\
&+\frac{3}{35} \sqrt{3} \sqrt{\frac{n+a}{3 a-n}}\left(3 a^{2} n+a^{3}+n^{3}+3 a n^{2}\right) n^{-2},
\end{aligned}
$$

and a similar result is now within reach, at least for specific values of $n$. 


\subsection{The First Hirzebruch Surface.}

Solutions to the Nonlinear Integrability Condition. Proceeding to the constraint $n<|k|$, again the case of positive $k$, i.e., the first Hirzebruch surface $(n=1)$, is given the more extensive treatment.

Theorem 4.3. Let $N=\mathbb{P}(L \oplus \mathcal{O})$ be a ruled surface constructed from a line bundle $L$ of Chern number one over a compact Riemann surface of genus zero or two. The momentum construction yields a family of central metrics of nowhere-vanishing non-constant central curvature in $N$. The sign of the latter is the same as the sign of the Euler number of the base.

For genus larger than one, the same methods should work for other choices of $k$ and $n$ in the realm $k<-n$.

Proof. We first discuss the case of genus zero. Analyzing the radicand, $f_{2}( \pm 1)=$ $\mp 4+5>0$. Since both $f_{2}$ and $f_{1}$ are positive in $(-1,1), h:=-f_{2} / f_{1}$ has a limit value equal to $-\infty$ at both endpoints. Therefore this function achieves a maximum at a point $\tau_{0}$ in the interior of $[-1,1]$. Again denoting by $b=b_{1}\left(\tau_{0}\right)$, this maximum value gives a radicand vanishing at $\tau_{0}$ : i.e., $f_{1}\left(\tau_{0}\right) b+f_{2}\left(\tau_{0}\right)=0$. Note that $b$ is necessarily negative.

Substituting $b$ for $b_{1}$ in (4.4) defines $P_{b}(\tau)$ and then $\Psi_{b}=R^{-1} \circ P_{b}$. The notation $\tau_{0}$ corresponds to the one given in Theorem 3.6 since $\left[\left(R^{\prime} \circ \Psi_{b}\right) / Q\right]\left(\tau_{0}\right)=0$, but one should keep in mind that the function $\Psi_{b}$ does not give rise to a central metric, and in fact its image contains $-k / n=-2$, which is outside the interval $\left[-d_{\infty}, d_{0}\right]$.

Proceeding to estimate $b$, one may note as in the previous subsection that $\tau_{0}$ is a critical point of $h(\tau)$. However, this gives in general an equation of degree four. A simpler relation can be obtained by noticing that $\tau_{0}$, as a minimum, is also a critical point of $f_{1}(\tau) b+f_{2}(\tau)=(2 / n) P_{b}(\tau)+(k / n+1)^{2}$. Substituting $b$ for $b_{1}$ in (4.3) and denoting the left-hand side by $b_{0}\left(\tau_{0}\right)$, it follows that $\tau_{0}$ is a zero of $P_{b}^{\prime}(\tau)=-\left(b_{0}\left(\tau_{0}\right)+b \tau\right)(a-n \tau)$, using (4.2). Since $a-n \tau>0$, one has, using (4.3) again (as well as the non-vanishing of $b$ ),

$$
\tau_{0}=\frac{-b_{0}\left(\tau_{0}\right)}{b}=-\frac{n}{3 a}-\frac{k}{a b}=-\frac{1}{3 a}-\frac{2}{a b},
$$

or

$$
b=-\frac{2}{a \tau_{0}+\frac{1}{3}}=-\frac{6}{3 a \tau_{0}+1} .
$$

Here the denominator cannot vanish since in the previous expression $2 /(a b) \neq 0$. For making the estimate on $b$, and then $I(b)$, it will be enough to use $-1<\tau_{0}<1$. Since $b$ is negative, from the right-hand inequality one deduces

$$
b<-\frac{6}{3 a+1} \text {. }
$$

In bounding the summand of $I(b)$ containing the radical, the relations

$$
\begin{aligned}
f_{2}(\tau) /(a-\tau)^{2} & =\left(5-4 \tau+2\left(\tau^{2}-1\right) / a\right) /(a-\tau)^{2} \\
& =2 / a+(5-2(a+1 / a)) /(a-\tau)^{2} \\
& \leq 2 / a+(5-2(a+1 / a)) /(a+1)^{2}=9 /(a+1)^{2}
\end{aligned}
$$


for $-1<\tau<1$ and $a \geq 2$, and $\sqrt{1-x}<1-x / 2$ for $x>0$, will be employed. The latter is used with $x:=-f_{1}(\tau) b / f_{2}(\tau)>0$. Restricting to $a \geq 2$ :

$$
\begin{aligned}
\int_{-1}^{1}( & a-n \tau) \sqrt{f_{1}(\tau) b+f_{2}(\tau)} d \tau \\
& =\int_{-1}^{1}(a-\tau) \sqrt{f_{2}(\tau)} \sqrt{1-\left(-\frac{f_{1}(\tau) b}{f_{2}(\tau)}\right)} d \tau \\
& \leq \int_{-1}^{1}(a-\tau) \sqrt{f_{2}(\tau)}\left(1-\left(-\frac{f_{1}(\tau) b}{2 f_{2}(\tau)}\right)\right) d \tau \\
& =\int_{-1}^{1}(a-\tau) \sqrt{f_{2}(\tau)} d \tau+\frac{b}{2} \int_{-1}^{1} \frac{f_{1}(\tau)}{\sqrt{f_{2}(\tau) /(a-\tau)^{2}}} d \tau \\
& <\int_{-1}^{1}(a-\tau) \sqrt{f_{2}(\tau)} d \tau-\frac{3}{3 a+1} \frac{a+1}{3} \int_{-1}^{1} f_{1}(\tau) d \tau \\
& =\frac{13 a}{3}-\frac{3}{(3 a+1)} \frac{a+1}{3} \frac{4}{3}\left(a-\frac{1}{3 a}\right)=\frac{1}{9} \frac{4 a+4+105 a^{3}+27 a^{2}}{a(3 a+1)} .
\end{aligned}
$$

Subtracting $4 a=\frac{2 a k}{n}$ from this expression gives

$$
-\frac{1}{9} \frac{9 a^{2}-4 a+3 a^{3}-4}{a(3 a+1)}
$$

which is negative for $a \geq 2$.

Since $I(b)<0<I(0)$, it follows from the previously mentioned continuity argument that there is a value of $b_{1}$ in the range

$$
b<-\frac{6}{3 a+1}<b_{1}<0,
$$

for which the right-hand side of (4.7) equals $2 a k / n$, i.e., a value for which the nonlinear integrability condition (4.7) is satisfied, for Kähler classes as above.

Positivity of the Central Curvature. The above estimate also proves that the solutions found have positive central curvature. To see this, first note that if $C(\tau)=0$ at some point in the interval, then $\tau=-b_{1} / b_{0}$ is a critical point of $P(\tau)$. So it must satisfy equation (4.8), and therefore the estimate (4.9), with $b_{1}$ replacing $b$. But since it was shown above that $b_{1}>-6 /(3 a+1)$, the central curvature does not vanish on $[-1,1]$. Its positivity then follows from an earlier remark, since the base has positive central curvature. Alternatively, its minimum value is, since $b_{1}<0$, equal to its value at $\tau=1$, which is $b_{0}+b_{1}=\left((3 a+1) b_{1}+6\right) /(3 a)>0$, by (4.3) and (4.11). Hence it is positive. By Theorem 3.7. the Ricci tensor of $g_{\varphi}$ is positive definite.

The Case of a Base of Genus $p>1$. When $k<0$ and $|k|=-k>n$, then

$$
\begin{aligned}
& f_{1}(\tau)=\frac{2}{n}\left(\tau^{2}-1\right)\left(\frac{n}{3} \tau+\left(\frac{n^{2}}{6 a}-\frac{a}{2}\right)\right), \\
& f_{2}(\tau)=\frac{k}{a} \tau^{2}-\frac{2 k}{n} \tau-\frac{k}{a}-\frac{2 k}{n}+\left(\frac{k}{n}+1\right)^{2},
\end{aligned}
$$


and the latter is increasing in $[-1,1]$. The equation replacing (4.8) is

$$
b=-\frac{k}{a \tau_{0}+\frac{n}{3}}=-\frac{3 k}{3 a \tau_{0}+n} .
$$

The positivity of the $f_{i}$ 's shows that the sign of the minimum $b_{1}:=b$ for which $f_{1}(\tau) b_{1}+f_{2}(\tau) \geq 0$ is still negative (though now $I$ has a maximum at $b$ ). Therefore $3 a \tau_{0}+n<0$, and one uses the negative bound $-1<\tau_{0}$ to get the bound

$$
b<-\frac{3 k}{n-3 a} .
$$

Concentrating on the case $n=1$, the expression comparable to (4.10) is

$$
-\left(\int_{-1}^{1}(a-\tau) \sqrt{f_{2}(\tau)} d \tau+\frac{b}{2} \int_{-1}^{1} f_{1}(\tau) / \sqrt{f_{2}(\tau) /\left(a-\tau^{2}\right)} d \tau+2 a k\right) .
$$

Notice that $2 a k$ was added and not subtracted to accommodate the sign change of the nonlinear integrability condition for $k<0$. For particular values of $k$ this can be evaluated. For example, when $k=-2$, one gets

$$
\frac{1}{9} \frac{15 a^{3}-21 a^{2}-8 a+8}{a(3 a-1)}
$$

which is positive even in a larger range than $a \geq 2$. Since this is opposite the sign of $I(0)$, the solution metric thus exists, and has $b_{1}>-3 k /(1-3 a)$. The central curvature is nowhere vanishing by the same argument as before. This conclusion and the sign could be found by computing its maximum value. This occurs at $\tau=-1$, and equals $b_{0}-b_{1}=\left((1-3 a) b_{1}+3 k\right) /(3 a)<0$. Therefore the central curvature is negative. This completes the proof of the theorem.

4.4. The Genus One Base. The remaining case is $|k|<n$, which involves patching the two branches of $\Psi$. These meet at a point $\tau_{0} \in(-1,1)$ whose properties are described in Theorem 3.6. Providing an estimate for this case is generally more difficult, since both $\tau_{0}$ and $b$ appear in the nonlinear integrability condition (3.9). This section treats the case $k=0$, in which $\tau_{0}$ (and $b$ ) can be evaluated explicitly.

Theorem 4.4. Over a compact manifold $M$ of genus one, the momentum construction yields no central metrics with non-constant central curvature on any space $N=\mathbb{P}(L \oplus \mathcal{O}) \rightarrow M$

Proof. The first simplification occurs since $f_{2}(\tau) \equiv 1$ identically when $k=0$. Next, from 4.3,

$$
\tau_{0}=-\frac{b_{0}\left(\tau_{0}\right)}{b}=-\frac{n}{3 a} .
$$

In other words, the vanishing of the Euler number $k$ implies that one has an expression for $\tau_{0}$ that does not depend on $b$. On the other hand, since $b$ is also the maximum of $h=-f_{2} / f_{1}$, which occurs at $\tau_{0}$, it evaluates to

$$
b=-\frac{1}{f_{1}\left(\tau_{0}\right)}=-81 \frac{n a^{3}}{\left(9 a^{2}-n^{2}\right)^{2}} .
$$


Therefore one needs to evaluate the integral

$$
\begin{aligned}
& I_{+}(b)+I_{-}(b)=\left(\int_{-1}^{\tau_{0}}-\int_{\tau_{0}}^{1}\right)(a-n \tau) \sqrt{1+f_{1}(\tau) b} d \tau \\
= & \left(\int_{-1}^{-\frac{n}{3 a}}-\int_{-\frac{n}{3 a}}^{1}\right)(a-n \tau) \sqrt{\frac{\left(9 a^{2}-6 n \tau a+n^{2}\right)(n+3 a \tau)^{2}}{\left(9 a^{2}-n^{2}\right)^{2}}} d \tau \\
= & \left(\int_{-1}^{-\frac{n}{3 a}}-\int_{-\frac{n}{3 a}}^{1}\right)(a-n \tau) \frac{|n+3 a \tau|}{\left(9 a^{2}-n^{2}\right)} \sqrt{\left(9 a^{2}-6 n \tau a+n^{2}\right)} d \tau .
\end{aligned}
$$

Just as in the case of the second Hirzebruch surface, this integral can be computed, and the result is

$$
-\frac{2}{105} \frac{n\left(n^{4}-42 a^{2} n^{2}+105 a^{4}\right)}{(n-3 a) a^{2}(n+3 a)} .
$$

The bracketed term of the numerator is larger than $n^{4}-42 a^{4}+105 a^{4}=n^{4}+63 a^{4}$ and so is never zero. Thus $I_{+}(b)+I_{-}(b) \neq 0$, and by the general argument for the 2-branch situation, as described at the end of section 4.1, the nonlinear integrability condition is never satisfied.

\section{REFERENCES}

[C0] E. Calabi, Métriques Kähleriennes et fibrés holomorphes, Annales Scientifiques de l'École Normale Supérieure 12 (1979), 268-294. MR 83m:32033

[C1] E. Calabi, Extremal Kähler metrics, Seminar on Differential Geometry (S. T. Yau, ed.), Annals of Mathematics Studies 102, Princeton University Press, Princeton, N.J., 1982, 259-290. MR 83i:53088

[C2] E. Calabi, Extremal Kähler metrics II, Differential Geometry and Complex Analysis (I. Chaval and H. M. Farkas, eds.), Springer, Berlin-New York, 1985, 95-114. MR 86h:53067

[Dr] A. Derdzinski, Self-dual Kähler manifolds and Einstein manifolds of dimension four, Compositio Math. 49 (1983), 405-433. MR 84h:53060

[FMS] A. Futaki and T. Mabuchi and Y. Sakane, Einstein-Kähler metrics with positive Ricci curvature, Kähler Metrics and Moduli Spaces, Advanced Studies in Pure Mathematics 18-II, Academic Press, Boston, MA, 1990, 11-83. MR 94c:32019

[G] Z.-D. Guan, Existence of extremal metrics on compact almost homogeneous Kähler manifolds with two ends, Trans. Amer. Math. Soc. 347 (1995), 2255-2262. MR 96a:58059

[H] A. D. Hwang, On existence of Kähler metrics with constant scalar curvature, Osaka J. Math. 31 (1994), 561-595. MR 96a:53061

[HSm1] A. D. Hwang and S. R. Simanca, Distinguished Kähler metrics on Hirzebruch surfaces, Trans. Amer. Math. Soc. 347 (1995), 1013-1021. MR 95e:58045

[HSm2] A. D. Hwang and S. R. Simanca, Extremal Kähler metrics on Hirzebruch surfaces which are locally conformally equivalent to Einstein metric, Math. Ann. 309 (1997), 97-106. MR 98f:58056

[HSn] A. D. Hwang and M. Singer, A momentum construction for circle-invariant Kähler metrics, Trans. Amer. Math. Soc. 354 (2002), 2285-2325.

[Ki] N. Koiso, On rotationally symmetric Hamilton's equation for Kähler-Einstein metrics, Recent Topics in Differential and Analytic Geometry, Advanced Studies in Pure Mathematics 18-I, Academic Press, Boston, MA, 1990, 327-337. MR 93d:53057

[KS1] N. Koiso and Y. Sakane, Nonhomogeneous Kähler-Einstein metrics on compact complex manifolds, Curvature and Topology of Riemannian Manifolds (Katata, 1985), Lecture Notes in Mathematics 1201, Springer, Berlin-New York, (1986), 165-179. MR 88c:53047

[KS2] N. Koiso and Y. Sakane, Nonhomogeneous Kähler-Einstein metrics on compact complex manifolds II, Osaka J. Math. 25 (1988), 933-959. MR 90e:53061 
[Le] C. LeBrun, Einstein metrics on complex surfaces, Geometry and Physics (Aarhus, 1995), Lecture Notes in Pure and Applied Mathematics, 184, Marcel Dekker, New York, 1997, 167-176. MR 97j:53048

[LSn] C. LeBrun and M. Singer, Existence and deformation theory for scalar-flat Kähler metrics on compact complex surfaces, Invent. Math. 112 (1993), 273-313. MR 94e:53070

[Mb] T. Mabuchi, Einstein-Kähler forms, Futaki invariants and convex geometry on toric Fano manifolds, Osaka J. Math 24 (1987), 705- 737. MR 89e:53074

[Ms1] G. Maschler, Distinguished Kähler metrics and Equivariant Cohomological Invariants, thesis, State University of New York at Stony Brook, August 1997.

[Ms2] G. Maschler, Central Kähler metrics, Trans. Amer. Math. Soc. 355 (2003), 2161-2182.

[PP] H. Pedersen and Y. S. Poon, Hamiltonian constructions of Kähler-Einstein metrics and Kähler metrics of constant scalar curvature, Comm. Math. Phys. 136 (1991), 309-326. MR 92f:32051

[Sk] Y. Sakane, Examples of compact Einstein Kähler manifolds with positive Ricci tensor, Osaka J. Math. 23 (1986), 585-616. MR 87k:53111

[Sm] S. R. Simanca, A note on extremal metrics of nonconstant scalar curvature, Israel J. Math. 78 (1992), 85-93. MR 93k:58054

[Tf] C. W. Tønnesen-Friedman, Extremal Kähler metrics on minimal ruled surfaces, J. Reine Angew. Math. 502 (1998), 175-197. MR 99g:58026

Department of Mathematics and Computer Science, College of the Holy Cross, WorCester, MASSACHUSETtS 01610

E-mail address: ahwang@mathcs.holycross.edu

Department of Mathematics, University of Toronto, Toronto, Ontario, Canada M5S 3G3

E-mail address: maschler@math.toronto.edu 